\title{
Adoptive immunotherapy of prostate cancer bone lesions using redirected effector lymphocytes
}

\author{
Jehonathan H. Pinthus, ${ }^{1,2}$ Tova Waks, ${ }^{1}$ Victoria Malina, ${ }^{1}$ Keren Kaufman-Francis, ${ }^{1}$ \\ Alon Harmelin, ${ }^{3}$ Itzhak Aizenberg, ${ }^{4}$ Hannah Kanety, ${ }^{5}$ Jacob Ramon, ${ }^{2}$ and Zelig Eshhar ${ }^{1}$ \\ 'Department of Immunology, The Weizmann Institute of Science, Rehovot, Israel. ${ }^{2}$ Department of Urology, Sheba Medica Center, Tel Hashomer, \\ Israel. ${ }^{3}$ Department of Veterinary Resources, The Weizmann Institute of Science, Rehovot, Israel. "Koret School of Veterinary Medicine, \\ Hebrew University, Jerusalem, Israel. ${ }^{5}$ Institute of Endocrinology, Sheba Medical Center, Tel Hashomer, Israel.
}

\begin{abstract}
Prostate cancer is currently the most commonly diagnosed noncutaneous malignancy in American men. When metastatic, usually to the bone, the disease is no longer curable and is usually treated palliatively with androgen ablation. However, after conversion to androgen-independent disease, there is no effective therapy currently available. The "T body" approach, which uses genetically reprogrammed lymphocytes derived from the patient and expressing chimeric receptor genes, combines the effector functions of T lymphocytes and NK cells with the ability of antibodies to recognize predefined surface antigens with high specificity and in a non-MHC-restricted manner. We show here the therapeutic efficacy of human lymphocytes bearing erbB2-specific chimeric receptors on human prostate cancer BM lesions in a SCID mouse model after conditioning of the recipient to allow homing and persistent functioning of the adoptively transferred cells. Induction of stromal cell-derived factor-1 production within the BM using low-dose irradiation or cyclophosphamide combined with IL-2 administration enhanced the homing of systemically delivered $T$ bodies, resulting in decreased tumor growth and prostate-specific antigen secretion, prolongation of survival, and even cure of the treated mice. These preclinical studies strongly support the idea that the $T$ body approach has therapeutic potential in disseminated prostate cancer.
\end{abstract}

\begin{abstract}
Introduction
Prostate cancer (PC) is the most commonly diagnosed malignancy and the second leading cause of cancer death in American men (1). Bone is the most common metastatic site for PC (2), and bone metastases are responsible for the majority of metastatic PC morbidity. Bone metastases are detected in more than $70 \%$ of patients who die of PC (3). Patients with metastatic PC have a median survival of only 30 months (4). After hormonal ablation therapy, currently the first standard treatment of these patients, a refractory state of hormone-independent disease usually develops within 12-18 months, after which approximately half of the patients die of disease progression within 6 months. Therefore, there is a great need to extend the repertoire of available therapeutic modalities against disseminated PC. The "T body" approach, in which effector lymphocytes are redirected with chimeric receptors with antibody specificity, has been suggested to hold promise for the adoptive immunotherapy of cancer (5-7). Recently, we demonstrated that human lymphocytes expressing chimeric receptors (CRs) (dubbed T bodies) specific for erbB2 can be directly applied to locally confined, well established PC xenografts, resulting in retardation of tumor growth, decrease in prostate-specific antigen (PSA) secretion, and prolongation of survival in the treated animals (8).
\end{abstract}

Nonstandard abbreviations used: $\mathrm{CR}$, chimeric receptor; PC, prostate cancer; PSA, prostate-specific antigen; scFv, single-chain variable region; SDF-1, stromal cell-derived factor-1; TBI, total body irradiation; TfR, transferrin receptor; TIL, tumorinfiltrating lymphocyte; TNP, 2,4,6-trinitrophenol.

Conflict of interest: The authors have declared that no conflict of interest exists.

Citation for this article: J. Clin. Invest. 114:1774-1781 (2004).

doi:10.1172/JCI200422284
Optimization of the $\mathrm{T}$ body approach for systemic therapy of advanced PC, however, must overcome several drawbacks before it can be applied clinically. One of the major limitations in the adoptive transfer of immune cells is the lack of specific trafficking of the genetically engineered $\mathrm{T}$ cells to sites of disseminated tumor. Trafficking of $\mathrm{T}$ cells to these sites is a complex multistage process involving rolling and arrest at specific sites along the endothelium, followed by extravasation and penetration of the target tissue. A cardinal step in these processes is mediated by the interaction between tissue-secreted chemokines and their corresponding receptors on the membranes of the T cells (9). Although tumor cells can secrete chemokines, site-specific trafficking of T cells does not generally occur in situations in which the $\mathrm{T}$ cells do not express the appropriate surface receptors for such chemokines (10).

Recently, Brentjens et al. (11) eradicated systemic B cell tumors in a mouse model using genetically targeted human $\mathrm{T}$ lymphocytes. In that system, human PBLs that were transduced with an anti-CD19-specific CR and then were activated and propagated in the presence of $\mathrm{CD} 19^{+} \mathrm{CD} 80^{+}$"artificial" antigen-presenting cells and IL-15 persisted in vivo and maintained their function for enough time to eliminate their distant tumor target.

Here we adopted lessons learned from human hematopoietic stem cell transplantation in order to attract $\mathrm{T}$ bodies to PC bone lesions in SCID mice. Our approach was based on the previous demonstration that human hematopoietic cells home and accumulate rapidly in the BM of SCID mice previously conditioned with total body irradiation (TBI) (12-14) or cyclophosphamide, treatments that increase stromal cell-derived factor-1 (SDF-1) expression in the BM (15). SDF-1, a member of the CXC subfamily of chemokines, is a potent chemoattractive factor for $\mathrm{T}$ and pre-B lymphocytes (16) 

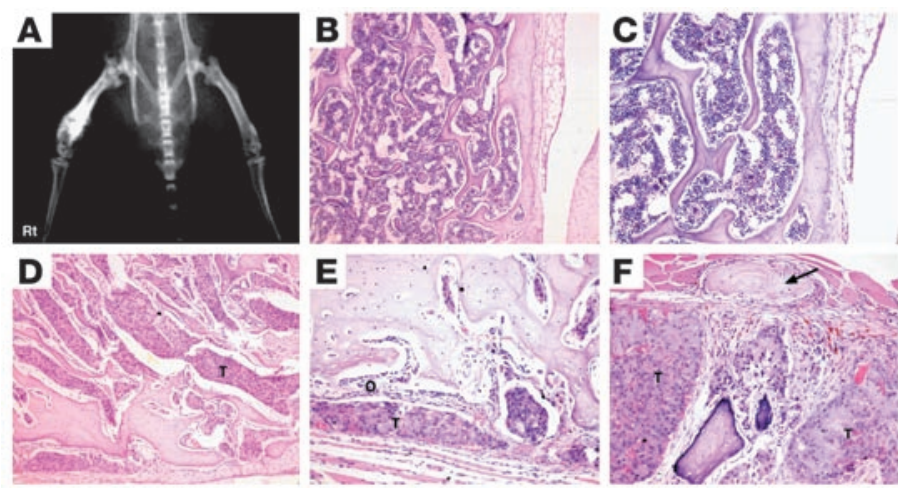

\section{Figure 1}

WISH-PC14 induces osteoblastic bone lesions. A single-cell suspension of WISH-PC14 was injected transtrochanterically into the right femur of a SCID mouse. (A) Radiographic appearance of the tumor. (B) Normal BM histology of the contralateral femur. Magnification, $\times 100$. (C) Same as B. Magnification, $\times 200$. Note the normal bone trabeculi and periosteum. (D) WISH-PC14 (T) occupies the entire BM cavity of the right femur. Magnification, $\times 100$. (E) Tumor cells surrounded by osteoblastic activity (O). Magnification, $\times 200$. Note the thickening of the bone trabeculi. (F) Same preparation and magnification as in $\mathbf{E}$. Note the periosteal reaction of paraosteal bone formation (arrow).

that express the SDF-1 receptor CXCR4 (17). SDF-1 affects T cell rolling and the strength of $\mathrm{T}$ cell adhesion to activated endothelial cells (18) as well as the costimulation of $\mathrm{CD}^{+}{ }^{+} \mathrm{T}$ cell activation (19).

Thus, we hypothesized that an increase in the concentration of SDF-1 within the microenvironment of the BM stroma, where PC bone metastases reside, would increase the homing and retention of systemically administered $\mathrm{T}$ bodies. Accordingly, we established an in vivo experimental system to extend the therapeutic scope of $\mathrm{T}$ bodies to metastatic cancer. We demonstrate here that treatments that induce SDF-1 expression in the BM enhance the homing of erbB2-specific human $\mathrm{T}$ bodies, resulting in the inhibition of advanced PC progression and even cure.

\section{Results}

Improving the migration of ex vivo-manipulated lymphocytes. We studied the ability of human $\mathrm{T}$ bodies to cure PC bone metastasis using an experimental, preclinical system in SCID mice bearing established human PC xenografts in the murine femur (Figure 1). These mice were systemically administered with either therapeutic (erbB2-specific) or control (2,4,6-trinitrophenol-specific

\section{Figure 2}

Effect of irradiation and cyclophosphamide on SDF-1 mRNA expression in $\mathrm{BM}$ and subsequent migration of human $\mathrm{T}$ bodies to murine BM. (A and B) SCID mice were irradiated with 2 Gy or injected i.p. with $200 \mathrm{mg} / \mathrm{kg}$ of cyclophosphamide or were left untreated. (A) Twenty-four hours after the treatments, mRNA was prepared and subjected to RT-PCR. Cycloph, cyclophosphamide; mSDF- $1 \alpha$, mouse SDF- $1 \alpha$; normal, untreated mouse. $\mathrm{H}_{2} \mathrm{O}$ was used as a negative control. (B) Kinetics of the migration of human $\mathrm{T}$ bodies to the BM after their i.v. injection into treated mice. The number of T bodies reaching the BM (events) was determined by FACS analysis gating on GFP ( $n=3$ mice per group; experiment was repeated at least twice). The number of T bodies that accumulated in the BM is significantly larger in irradiated than in nonirradiated recipients at 6 and 12 hours $(P=0.054$ and $P=0.011$, respectively).
[TNP-specific]) human T bodies. However, our first attempts to eliminate such bone metastasis failed, and we found almost no effect produced by the erbB2-specific $\mathrm{T}$ cells (data not shown). Because the same erbB2-specific T bodies were previously found to be very effective in the elimination of the same subcutaneous PC xenografts after their intratumoral administration (8), we suspected that the $\mathrm{T}$ bodies, after their i.v. administration, failed to reach (in sufficient quantities) and efficiently reject the bone tumors. The in vitro conditions used for activation of the human $\mathrm{T}$ cells and their culture in IL-2, which are required for retroviral transduction and proliferation (20), have been demonstrated to downregulate the surface expression of several molecules that are involved in the migration of lymphocytes, including CXCR4 $(17,21)$. We therefore studied whether increasing the levels of SDF-1, the CXCR4 ligand, might enhance the migration of $\mathrm{T}$ bodies to the $\mathrm{BM}$ and thereby eliminate bone tumors expressing the corresponding target antigen. Although the $\mathrm{T}$ bodies are of human origin and the SDF-1 is produced by the SCID mouse, there is almost complete identity and cross-reactivity between the SDF- 1 molecules of these 2 species $(22,23)$. Because SDF-1 is produced in response to tissue damage as part of the regenerative process (15), we tested the effects of treatments known to elevate its concentration in the bone, such as TBI and cyclophosphamide (15). Figure 2A shows that sublethal irradiation with a dose of 2 Gy resulted in an increase in SDF-1 expression in SCID BM. Cyclophosphamide $(200 \mathrm{mg} / \mathrm{kg}$, i.p.) also induced increasing levels of SDF-1 mRNA expression in the BM (Figure 2A). Accordingly, 2 Gy TBI or $200 \mathrm{mg} / \mathrm{kg}$ cyclophosphamide was used to precondition the mice before the systemic administration of CR-bearing human lymphocytes. Indeed, these engineered lymphocytes repopulated the murine BM to a greater extent after this conditioning protocol. The level of human $\mathrm{T}$ body migration to murine BM peaked within 12-24 hours of TBI (Figure 2B). Similar results were obtained with a single low dose of cyclophosphamide (data not shown).

To test whether the enhanced migration of the engineered $T$ cells to the BM was due to the production of SDF-1 after preconditioning with low-dose TBI or cyclophosphamide, we treated the T bodies with anti-CXCR4 or control antibodies, including a mAb spe-

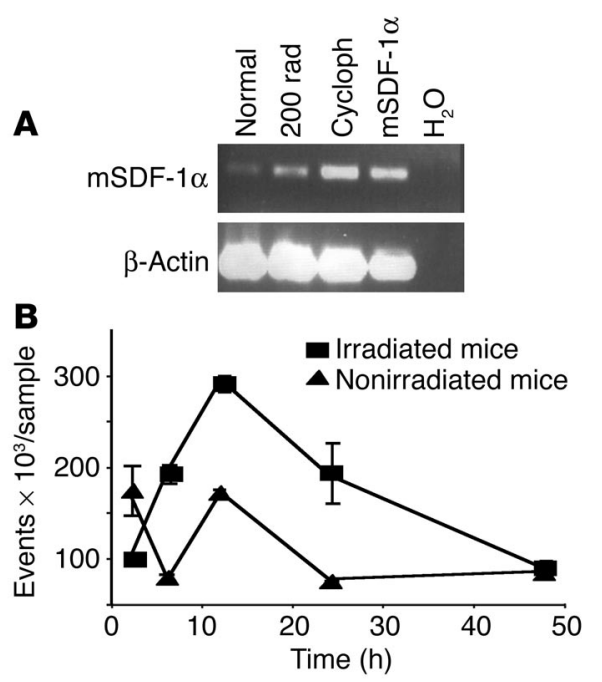




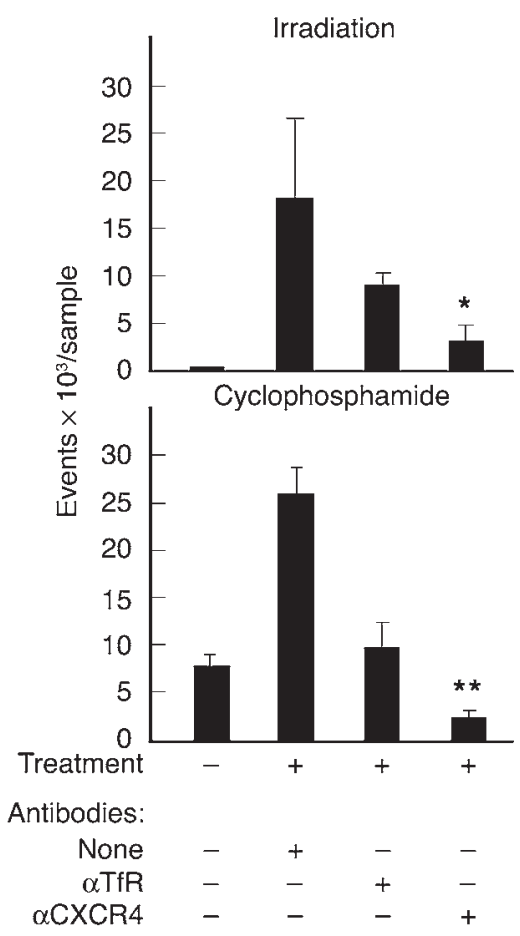

cific to the human transferrin receptor (TfR) (which is expressed on the lymphocyte surface and is of the same isotype as the mAb against CXCR4). The results depicted in Figure 3 show that the anti-CXCR4 partially yet significantly $(P=0.003$, compared with anti-TfR) inhibited the specific in vivo migration of the $T$ bodies. Similar results were obtained in a transwell migration assay, in which the migration of CR-bearing lymphocytes was specifically inhibited only by anti-CXCR4 (Figure 4).

The therapentic effect of systemically administered T bodies on PC BM lesions. After we established protocols for improving the trafficking to the bone of ex vivo-manipulated human lymphocytes within SCID mice, we tested the therapeutic efficacy of the erbB2-specific human lymphocytes. The results of systemic administration of $\mathrm{T}$ bodies into SCID mice bearing established intrafemoral PC (WISH-PC14) xenografts are shown in Figures 5-7. In this set of experiments, mice were either irradiated (2 Gy TBI) or left nonirradiated 24 hours before i.v. administration of erbB2- or TNP-specific human T bodies or medium alone. As demonstrated by all 3 endpoints, serum PSA (Figure 5), bone radiography (Figure 6), and animal survival (Figure 7), a specific anti-tumor effect was achieved only in the group treated with anti-erbB2 CR-bearing lymphocytes in mice that were preconditioned with low-dose TBI. There was no significant statistical difference between the groups of nonirradiated mice (Figure 5A); however, for the irradiated

\section{Figure 4}

Transwell migration of $\mathrm{T}$ bodies toward recombinant human SDF- $1 \alpha$ (rhSDF-1 $\alpha$ ) is dose dependent and is inhibited by anti-CXCR4. (A and B) Transwell migration of T bodies. (A) T bodies migrate toward increasing concentrations of rhSDF-1 $\alpha$. (B) T bodies were preincubated for 30 minutes on ice with anti-CXCR4 or anti-TfR prior to migration for 1 hour toward medium containing rhSDF- $1 \alpha(150 \mathrm{ng} / \mathrm{ml})$ or medium alone. Anti-CXCR4 significantly inhibited migration compared with anti-TfR or no treatment $\left({ }^{*} P=0.016\right.$ and ${ }^{*} P=0.019$, respectively).

\section{Figure 3}

Migration of T bodies to the BM of preconditioned mice is mediated in part by CXCR4. SCID mice ( $n=3$ mice per group) were irradiated with 2 Gy TBI or were injected with $200 \mathrm{mg} / \mathrm{kg}$ cyclophosphamide, 24 hours before i.v. administration of erbB2-specific CR-bearing lymphocytes. $T$ bodies were preincubated for 30 minutes on ice with $\mathrm{mAb}$ against CXCR4 ( $\alpha$ CXCR4), irrelevant control mAb against human TfR ( $\alpha$ TfR), or no antibody (none) and then were injected systemically into the mice. After 24 hours, BM was extracted and the number of human lymphocytes was measured by FACS analysis (detecting GFP-positive $T$ bodies in the irradiation experiment and CFSE-labeled cells in the cyclophosphamide experiment). Treatment with anti-CXCR4 was significantly different from control treatment (anti-TfR) for both irradiated mice $\left({ }^{\star} P=0.003\right)$ and cyclophosphamide-treated mice $\left({ }^{* *} P=0.009\right)$ and was significantly different from no antibody treatment ( $P=0.01$ and $P=0.0002$, respectively).

mice, the difference between the group treated with erbB2-specific $\mathrm{T}$ bodies and either the group treated with TNP-specific T bodies or the nontreated group (given medium alone; Figure $5 \mathrm{~B}$ ) was significant $(P=0.0145$ or $P=0.0455$, respectively). No therapeutic effect was induced by irrelevant $T$ bodies (TNP-specific) or in nonirradiated mice. Sublethal TBI only slightly decreased the rate of WISH-PC14 xenograft growth in the BM (as evident by comparison of the growth of control [medium-treated] xenografts in nonirradiated versus irradiated mice).

To test if this approach was also effective when the tumor-bearing mice were preconditioned with cyclophosphamide instead of irradiation, and also to test these effects on another tumor xenograft, we used LuCaP 35, an androgen-independent and erbB2-expressing PC xenograft. In this xenograft model, a weak yet significant $(P=0.037)$ decrease in tumor growth (as evidenced by PSA secretion) was seen only during the first month of the experiment in the preirradiated group treated with erbB2-specific T bodies (Figure 8). However, pretreatment with cyclophosphamide (Figure 9) caused a more pronounced and persistent therapeutic effect of the erbB2-specific $T$ bodies compared with that of control cells of irrelevant (TNP) specificity $(P=0.011)$. In addition, $35 \%$ ( 4 of 11 ) of mice in the group treated with
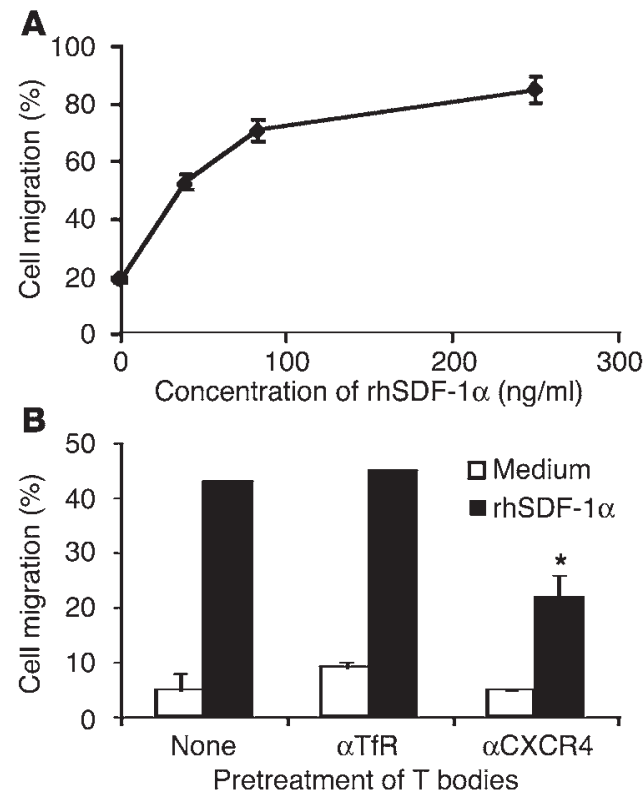


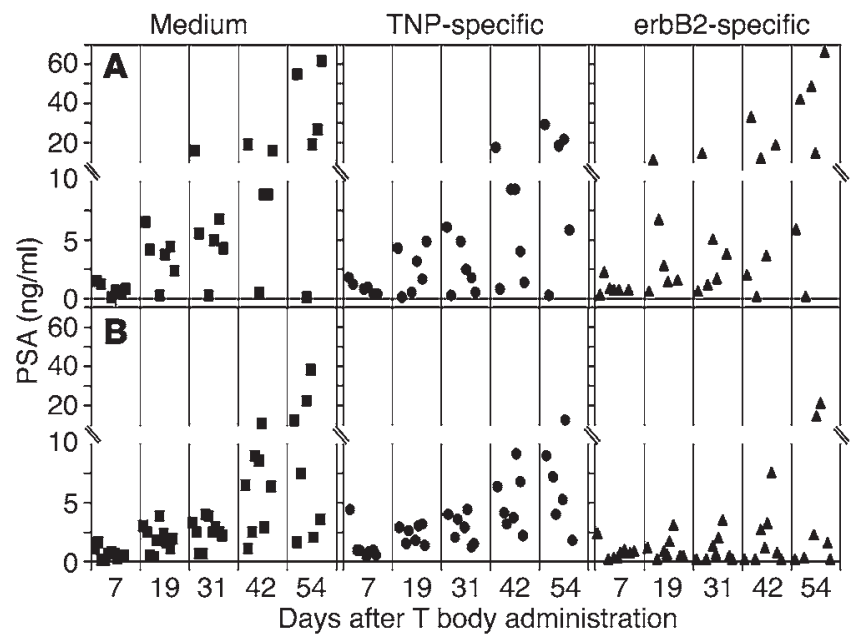

the erB2-specific T bodies maintained low serum levels of PSA $(<5 \mathrm{ng} / \mathrm{ml})$ for as long as 2 months after treatment.

\section{Discussion}

The development of genetically modified $\mathrm{T}$ cells expressing chimeric receptors specific to preselected tumor antigens has overcome several critical obstacles to adoptive cellular immunotherapy. Clinically relevant amounts of tumor-specific, autologous lymphocytes bearing CRs of various subtypes can now be produced $(8,11,20)$. These cells, which are specific to the same non-MHC and restricted antigen, are capable of working in concert upon stimulation by their predefined target. A large repertoire of CRs that can selectively recognize different tumor-associated antigens has been constructed $(10,24-39)$ and in practice one can attack a given tumor with a panel of $\mathrm{T}$ bodies including several different specificities and CR designs. This arsenal should offer a workable solution to one of the major drawbacks of cancer immunotherapy: the regrowth of escape variants as a result of selective immunological pressure $(7,40,41)$. Likewise, tripartite CRs, such as those used in these studies, which have additional costimulatory functions such as CD28 and 4-1BB $(20,42,43)$, which confer on the programmed $\mathrm{T}$ cells the desired features to offset the anergy and apoptotic signals often noted in tumor-bearing hosts (7).

One of the obstacles in the clinical application of $\mathrm{T}$ bodies that has been observed in both preclinical models and initial clinical trials is the inefficient homing of the ex vivo-manipulated $\mathrm{T}$ bodies to their tumor targets. Apparently, PBL-derived gene-modified T cells, after their activation, transduction, and prolonged propagation ex vivo, alter their homing properties and do not reach sites of disseminated disease in effective amounts, thereby failing to persistently maintain their effector function. This pattern is general and unrelated to CR expression, since non-gene-modified tumor-

\section{Figure 6}

Radiography of BM lesions after treatment. Radiographic analysis of mice 5 weeks after treatment. While tumor is noted in the irradiated and nonirradiated control groups of mice as well as in the nonirradiated animals treated with erbB2-specific $T$ bodies, in the irradiated animals treated with erbB2 CR-bearing lymphocytes, no radiographic signs of tumor are evident 5 weeks after preirradiation and treatment with erbB2-specific $\mathrm{T}$ bodies.

\section{Figure 5}

The effect of systemic treatment with erbB2-specific $T$ bodies on WISH-PC14 BM lesions is dependent on the preirradiation of mice. (A and B) SCID mice bearing an established intraosseous WISH-PC14 xenograft either were not preconditioned $(\mathbf{A})$ or were preconditioned with 2 Gy TBI (B) 24 hours before i.v. administration of erbB2- or TNP-specific CR-bearing lymphocytes or medium ( $n=10$ mice per group). PSA levels in the mouse sera were determined. Squares, circles, and triangles represent medium, TNP-, and erbB2-specific CRbearing lymphocytes, respectively, injected into recipient SCID mice.

infiltrating lymphocytes (TILs) also repeatedly failed in clinical trials to demonstrate persistent and functional engraftment in patients in the absence of cytoablative treatment (44-46). One manifestation of this problem is the entrapment of systemically administered ex vivo-manipulated lymphocytes in the lungs of experimental animals (47). In attempt to overcome this major limitation, and in order to optimize the $\mathrm{T}$ body approach for systemic treatment of disseminated cancer, we have shown here that preconditioning of the recipient with low-dose TBI or cyclophosphamide improves the therapeutic outcome produced by adoptively transferred $\mathrm{T}$ bodies. Our data suggest that SDF-1-CXCR4 interactions

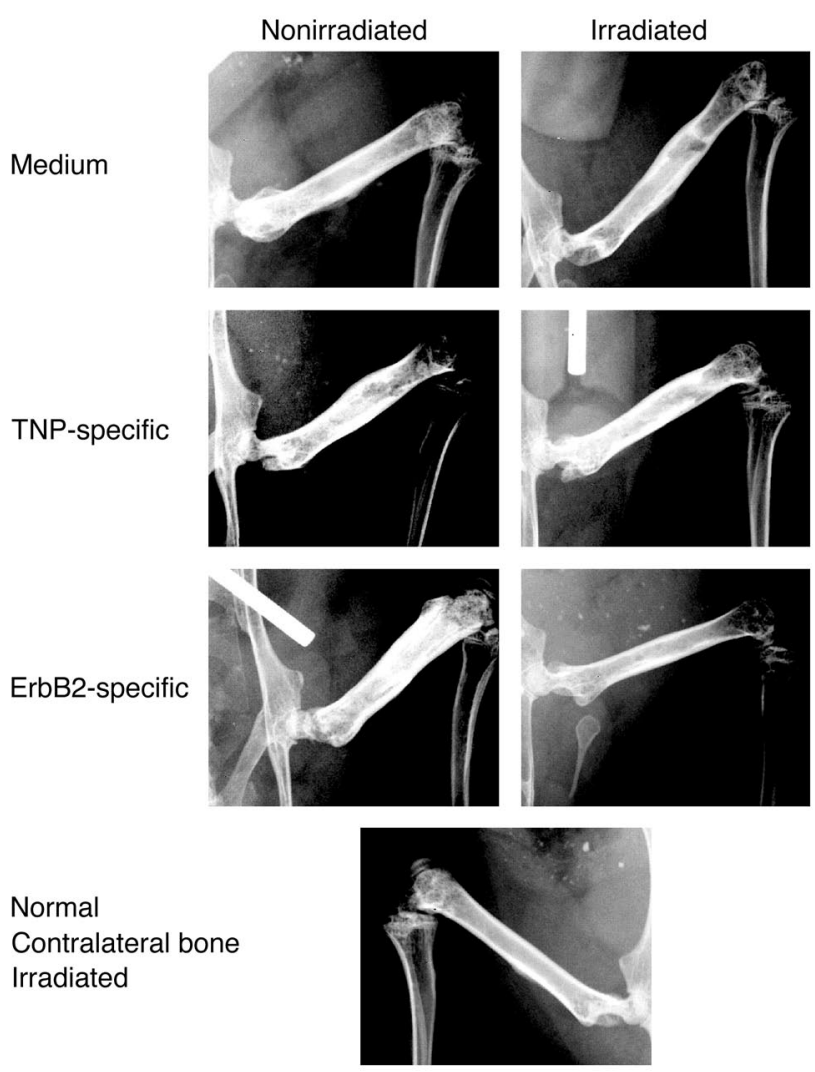




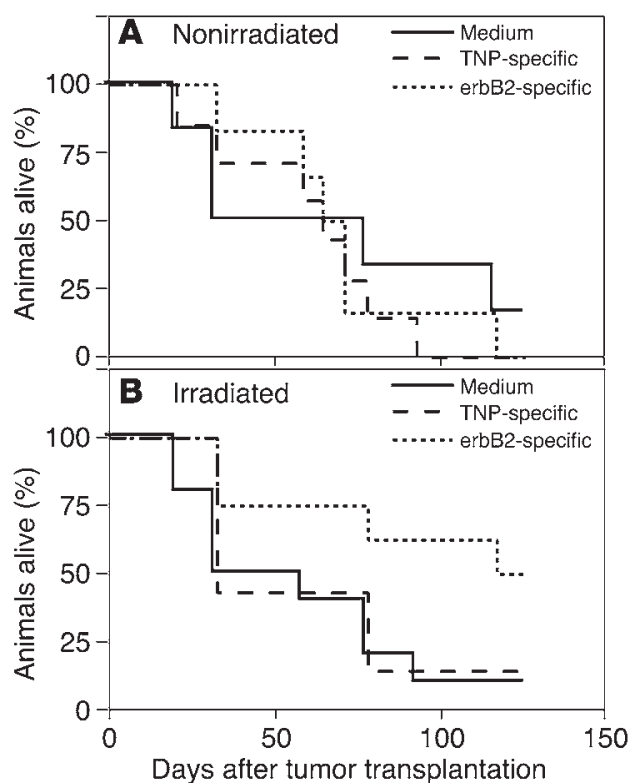

are involved in at least part of the effect. It is well documented that tissue-impairing agents (such as cyclophosphamide or TBI) induce the production and secretion of SDF-1 as part of the tissueregenerative process (15). SDF-1 is known to be a powerful mediator of the chemokinesis and chemotaxis of CXCR4-expressing hematopoietic stem cells $(14,23)$ as well as of mature lymphocytes such as NK and T cells $(17,21,48)$. As a model for an advanced and incurable tumor target suitable for $\mathrm{T}$ body immunotherapy, we have chosen to study PC bone metastasis. The BM is the principal site for the PC metastasis responsible for the vast morbidity and mortality from this disease. Of the several PC-associated antigens expressed on the cell surface (49-52), we elected to target erbB2, as it is overexpressed on up to $80 \%$ of metastatic PCs and its expression increases as androgen dependence decreases (53-55). Furthermore, erbB2 expression was suggested to be associated with the progression of $\mathrm{PC}(54,55)$. The two xenografts that we used in this preclinical study, WISH-PC14 (8) and LuCaP 35 (56), represent an aggressive phenotype of prostatic adenocarcinoma; WISH-PC14 is radiation resistant (data not shown), and LuCaP 35, which was established from a metastatic lymph node, grows independently of androgens (56) and is radiation resistant as well. Neither of these xenografts spontaneously metastasizes to the bone in SCID mice, yet they form typical PC bone lesions upon their injection into mouse BM (shown here for WISH-PC14 in Figure 1). The combined approach of recipient preconditioning plus adoptive transfer of tumor-specific $\mathrm{T}$ bodies was demonstrated to be effective for both PC xenografts (Figures 5-9).

Of the 2 pretreatments, cyclophosphamide was more effective in creating the proper conditions to enable the CR-bearing lymphocytes to exert their therapeutic effect in SCID mice. After low-dose cyclophosphamide treatment, a larger number of cells migrated to the $\mathrm{BM}$ (Figures 2 and 3). One explanation is that the 2 processes are interrelated; i.e., under the experimental conditions, cyclophosphamide induced more SDF-1 production in the BM, which attracted to the site more therapeutic $\mathrm{T}$ bodies. At the concentrations used, murine lymphocyte subpopulations differ in their response to cyclophosphamide. While effector and helper $\mathrm{T}$ cells are resistant, suppressor (or regulatory) $\mathrm{T}$ cells are sensitive (57). Yet in the

\section{Figure 7}

Systemic administration of erbB2-specific human lymphocytes prolongs the survival of mice bearing PC BM lesions. (A and B) Survival data of SCID mice bearing established WISH-PC14 bone lesions ( $n=10$ mice per group) systemically treated with TNP- or erbB2-specific CR-bearing lymphocytes or with medium, without (A) or with (B) preconditioning with 2 Gy TBI.

immune-deficient SCID mouse, such an effect, as well as an effect on lymphocytes homeostasis, is expected to be negligible.

Similar observations were made in a transgenic mouse model of a pancreatic islet cell tumor, in which higher doses of irradiation conditioned the tumor microenvironment by increasing the expression of adhesion molecules, cytokines, and chemokines (58). The authors suggested that radiation-induced changes to the endothelium reverse its nonadhesive phenotype and thus directly facilitate leukocyte-endothelium interactions. In this setting, combined irradiation/adoptive transfer induced the enhanced expression of IFN- $\gamma$, TNF- $\alpha$, and the angiostatic chemokines Mig and IP10. The fact that we used an immunodeficient host does not negate the possibility that such proinflammatory mechanisms are involved in our model as well. Indeed, Ganss et al. (58) showed that irradiation alone induced proinflammatory events, which did not reach a critical threshold required to activate the endogenous immunity after BM reconstitution, and that tumor rejection occurred only after subsequent infusion of activated effector cells. We believe that in normal, immunocompetent subjects, the overall effect of lymphoablative pretreatments, such as those used here, is complex and includes perturbation of the homeostasis to allow the grafting of exogenous lymphocytes, elimination of suppressor

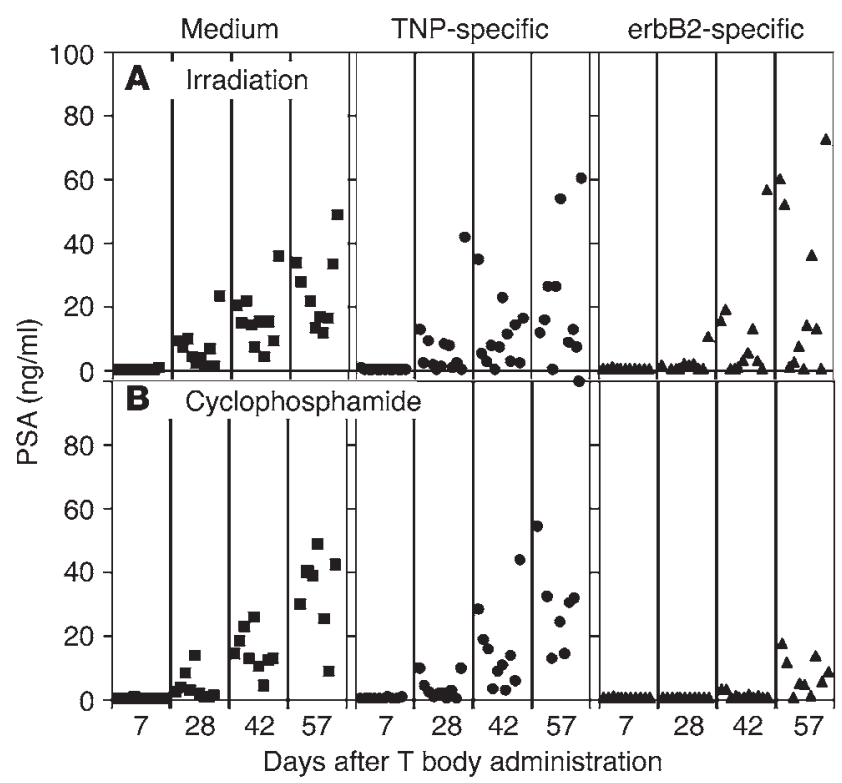

\section{Figure 8}

Pretreatment with cyclophosphamide or irradiation enhances the effect of systemic therapy with erbB2-specific CR-bearing lymphocytes on LuCaP-35 bone lesions. (A and B) SCID mice bearing established intraosseous LuCaP-35 xenografts were preconditioned with TBI (A) or cyclophosphamide (B) 24 hours before i.v. administration of erbB2-specific (triangles) or TNP-specific (circles) CR-bearing lymphocytes or medium (squares) ( $n=10$ mice per group). 


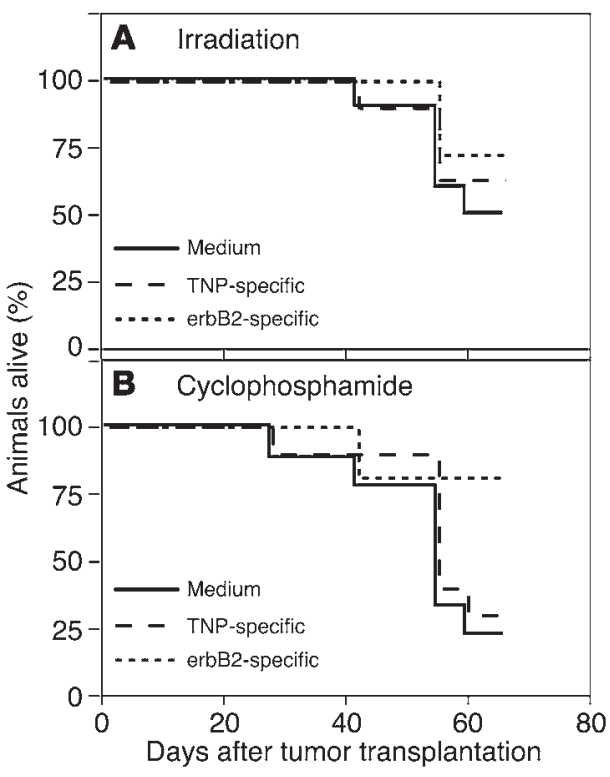

Figure 9

Effect of systemic therapy with erbB2-specific CR-bearing lymphocytes on the survival of mice with LuCaP-35 bone tumors. (A and B) Survival data of SCID mice bearing established LuCaP-35 bone tumors ( $n=10$ mice per group) systemically treated with TNP- or erbB2-specific CR-bearing lymphocytes or medium and preconditioned with 2 Gy TBI (A) or cyclophosphamide (B).

cells, and induction of an inflammatory milieu, which includes chemokines that attract $\mathrm{T}$ lymphocytes.

Based on the fact that anti-CXCR4 partially blocked $\mathrm{T}$ body migration to the BM (Figure 3) (as well as the migration of $\mathrm{T}$ bodies across an SDF-1 gradient in the transwell experiments; see Figure 4), and that both irradiation and cyclophosphamide increased SDF-1 expression, we believe that at least part of the therapeutic effect involves the CXCR4-SDF-1 interaction. SDF-1 plays a critical role not only in attracting hematopoietic progenitors into the BM $(12-14,18,23)$ but also in the migration of NK and NKT cells into the BM compartment (17). Moreover, several studies reported the activation of $\mathrm{T}$ cells by SDF-1: it serves as costimulatory factor for $\mathrm{CD}^{+} \mathrm{T}$ cell activation (19), and its secretion at the tumor site (BM in this case) enhances the development of a long-lived, tumor-specific cytotoxic T lymphocyte response (59), resulting in rejection of tumors. Recently, it was demonstrated by Dudley et al. that nonmyeloablative conditioning of metastatic melanoma patients with cyclophosphamide and fludarabine, before the adoptive transfer of tumor-reactive $\mathrm{T}$ cells, is essential for the persistent repopulation of clones of functional lymphocytes and for their trafficking to metastatic sites. The use of this protocol led to complete tumor regression in a significant number of patients (60). Although the mechanism was not determined in that study, we suggest that an increase in SDF-1 expression was induced in those patients in a manner similar to that demonstrated here in SCID mice.

A theoretical concern regarding the intentional induction of SDF-1 in cancer patients is the potential to also elicit unwanted migration to the bone of cancer cells overexpressing CXCR4 (61). Although most cell lines derived from common solid human tumors do not express CXCR4 (62), involvement of the CXCR4-SDF-1 axis in the metastasis of breast cancer was recently reported (63). The mechanism of action of SDF-1 in the promotion of metastasis is complex. In a breast cancer model (63), anti-CXCR4 reduced metastasis by inhibiting the CXCR4-mediated enhancement of cancer cell invasion to the tissue. Similarly, in rhabdomyosarcoma (62) it was demonstrated that the effect of SDF-1 involved mainly cell interactions with the microenvironment and their migration rather than cell proliferation and survival. However, in a human colon cancer xenograft model (64), it was demonstrated that the activation of CXCR4 does not play an important role in invasion but instead is responsible for the outgrowth of micrometastasis.

Taken together, our results suggest that tumor-specific $\mathrm{T}$ bodies are attracted by SDF-1 to migrate to the BM, where they eradicate tumor cells. In patients, SDF-1 may be responsible for only part of the effect; nevertheless, our results and the recently published clinical study using TILs for melanoma immunotherapy (60) strongly justify the preconditioning of cancer patients with nontoxic, lowdose irradiation or cyclophosphamide and fludarabine prior to the adoptive transfer of tumor-specific $T$ cells.

\section{Methods}

Mice. All animal experiments were approved by the Weizmann Institute Animal Care and Use Committee. Male mice (CB.17-SCID BEIGE; Charles River) 7-10 weeks old were maintained at the Specific Pathogen Free (SPF) animal facility at the Weizmann Institute of Science.

Tumormodels. As model PC BM lesions, we used the WISH-PC14 xenograft (8) that we previously established from a late recurrent primary tumor after definitive radiation therapy and the LuCaP 35 xenograft (a kind gift from R.L. Vessella, University of Washington School of Medicine, Seattle, Washington, USA) $(56,65)$. Both lines overexpress erbB2, secrete PSA into the host blood, are radiation resistant, and induce osteolytic and osteoblastic responses (ref. 65 and Figure 1). For tumor induction, $1 \times 10^{6}$ tumor cells were inoculated directly into the femur by a transtrochanteric route. This surgical procedure and the i.p. mini-osmotic pump implantation that required laparotomy were performed in mice sedated by general anesthesia with ketamine plus xylazine (127.5 and $4.5 \mathrm{mg} / \mathrm{kg}$, respectively). In the case of WISH-PC14, SCID mice were transplanted s.c. with 90-day slow-release testosterone pellets (12.5 mg/pellet; Innovative Research of America) before tumor administration.

Antibodies. The N29 mAb against ErbB2 was used as a source of the scFv, single-chain variable region ( $\mathrm{scFv}$ ) for construction of the CR (66). Rabbit anti-N29 idiotype and mouse mAb against human TfR (clone V1-10) were prepared in our laboratory. SP6 scFv (20) (with anti-TNP specificity) was used as a control. The $12 \mathrm{G} 5 \mathrm{mAb}$ against human CXCR4 (R\&D Systems Inc.) was used as indicated in Figures 3 and 4.

$C R$ genes and their expression in human lymphocytes. The $C R$ genes used in this study were of the tripartite configuration in which the scFv was linked through the CD28 extracellular domain (from which the ligand-binding region was truncated) to the intracellular part of the FcRI $\gamma$ chain (20). CRs derived from 2 scFvs were used in this study: an erbB2-specific receptor derived from the $\mathrm{N} 29 \mathrm{mAb}$ and, as a control, a TNP-specific one made from the Sp6 mAb. The CR cDNA construct was cloned into the pBullet vector followed by an internal ribosome entry site sequence and the GFP gene (20). Transduction of PBLs from healthy donors, preactivated with $\mathrm{mAb}$ against $\mathrm{CD} 3$ plus $\mathrm{mAb}$ against $\mathrm{CD} 28$, was performed with vector-containing supernatants in the presence of IL-2 on RetroNectin (FN-Takara Shuzo Ltd.) as described previously (20).

Treatment of PC xenografts in SCID mice. Mice bearing established tumors, with similar tumor load (as determined by serum PSA levels), were randomly assigned to different experimental groups (usually 7-10 days after tumor injection). In experiments in which continuous systemic adminis- 
tration of IL-2 was used, Alzelt mini-osmotic pumps (Durcet Corp.) were transplanted i.p. Each pump was loaded with recombinant human IL-2 (R\&D Systems Inc.) and was set to continuously release 105 units/h for 1 week. At that time, the mice were irradiated ( 2 Gy TBI from a cobalt source) or received cyclophosphamide $(200 \mathrm{mg} / \mathrm{kg}$ i.p.) or were not preconditioned by either of those methods. After 24 hours, $5 \times 10^{7}$ CR-bearing lymphocytes ( $\mathrm{T}$ bodies) were injected into the tail vein. As a control, medium alone (HBSS) was injected similarly to allow monitoring of the natural history of the disease under the same experimental conditions. Follow-up included periodic serum PSA determination, serial X-ray documentation of the bone lesions, and monitoring of the survival of the animals. Mice with a high tumor burden were euthanised.

Semiquantitative RT-PCR. Total RNA was isolated from mouse BM using the TRI Reagent kit (Molecular Research Center Inc.) according to the manufacturer's protocol. Each RNA sample $(1 \mu \mathrm{g})$ was subjected to cDNA synthesis for 1 hour at $42^{\circ} \mathrm{C}$ using the Reverse Transcription System kit (Promega Corp.). PCR was performed in 50- $\mu$ l reaction mixture containing $5 \mu \mathrm{l}$ of cDNA, $1 \mu \mathrm{l}$ of dNTP mixture (10 mM; Promega Corp.), primers for mouse SDF- $1 \alpha$ (5'-GGACGCCAAGGTCGTCGCCGTG-3' and 3'-TTGCATCTCCCACGGATGTCAG-5'; 335 bp), $5 \mu$ of $10 \times \mathrm{NH}_{4}$ buffer, $1.5 \mu \mathrm{l}$ of the $50-\mathrm{mM} \mathrm{MgCl}_{2}$ solution, and 5 units/reaction sample of BIOTAQ DNA polymerase (UK Bioline Ltd.). The quality of all cDNA samples was confirmed by PCR using primers for mouse $\beta$-actin ( $5^{\prime}$-TCCTGTGGCATCCATGAAACTACATTCAATTCC-3' and $3^{\prime}$-GTGAAAACGCAGCTCAGTAACAGTCCGCCTAG-5'; 347 bp). Semiquantitative analysis of mSDF- $1 \alpha$ expression was accomplished with 25 cycles of PCR at an annealing temperature of $65^{\circ} \mathrm{C}$ for 45 seconds.

FACS analysis. Specific homing to the BM was evaluated by FACS analysis of BM-derived cells by gating on green fluorescence emitted from the GFP-positive CR-bearing human lymphocytes. To avoid bias derived from the effect of TBI or cyclophosphamide, which decreases the number of murine cells and thereby increases the percentage of the transduced lymphocytes, we adjusted all BM cell preparations to the same volume, and the number of the human cells (gated by GFP expression) counted during a fixed time period.

Homing assay. For analysis of homing, $5 \times 10^{7}$ human $\mathrm{T}$ bodies were injected i.v. into mice 24 hours after TBI or cyclophospamide treatment, with or without a continuous supply of IL-2. Where indicated (see Figure 3), cells were incubated for 30 minutes at $4^{\circ} \mathrm{C}$ with $\mathrm{mAb}$ against human CXCR4 or with control mAb against human TfR ( $3 \mu \mathrm{g} / 10^{6}$ cells $)$ and then were injected i.v. without removal of the antibodies. Mice were sacrificed at different time points, and BM cells were recovered and analyzed by FACS for the presence of human cells as detailed above. Cells obtained from the $\mathrm{BM}$ of untreated mice served as controls.

1. Jemal, A., et al. 2003. Cancer statistics, 2003 CA Cancer J. Clin. 53:5-26.

2. Yonou, H., et al. 2001. Establishment of a novel species- and tissue-specific metastasis model of human prostate cancer in humanized non-obese diabetic/severe combined immunodeficient mice engrafted with human adult lung and bone. Cancer Res. 61:2177-2182.

3. Whitmore, W.F., Jr. 1984. Natural history and staging of prostate cancer. Urol. Clin. North. Am. 11:205-220.

4. Hossain, N.H., and Crawford, D. 1997. Androgen deprivation strategies for metastatic prostate cancer. In Principles and practices of genitourniary oncology. D. Raghavan, H.I. Scher, S.A. Leibel, and P. Lange, editors. Lippincott-Raven. Philadelphia, Pennsylvania, USA. 591-597.

5. Eshhar, Z. 1997. Tumor-specific T-bodies: towards clinical application. Cancer Immunol. Immunother 45:131-136.
CFSE labeling of T bodies. T bodies were labeled with the intracellular fluorescent dye CFSE (C-1157; Molecular Probes) by incubation for 8 minutes at room temperature in the absence of serum at a density of $10^{7} \mathrm{cells} / \mathrm{ml}$ in $5 \mu \mathrm{M}$ CFSE. CFSE loading was stopped by the addition of an equal volume of cold FCS. Cells were washed twice in complete RPMI medium (67).

In vitro migration assay. Chemotaxis experiments with CR-transduced lymphocytes used Costar Transwells (6.5-mm diameter and 5-mm pore size; Corning Costar) as described (15). Chemotaxis buffer (RPMI 1640 medium and $1 \%$ FCS) containing $2 \times 10^{5}$ cells in a volume of $0.1 \mathrm{ml}$ was added to the upper chamber and $0.6 \mathrm{ml}$ of chemotaxis buffer with or without SDF-1 (R\&D Systems Inc.) at the indicated concentrations (see Figure 4) was added to the bottom chamber. Cells migrating within 1 hour to the bottom chamber of the transwell were counted under a microscope. Preincubation with antibodies was done as described for the homing assay.

Serum PSA determination. PSA levels in mouse serum were determined using two chemiluminescent immunometric assays (Immulite; Diagnostic Products Corp.): the third-generation PSA kit (LKUP1), with a sensitivity of 0.003 $\mathrm{ng} / \mathrm{ml}$, or the LKPS1 PSA kit, with a sensitivity of $0.03 \mathrm{ng} / \mathrm{ml}$.

Statistical analysis. Statistical analysis was performed using JMP statistical software (SAS Institute Inc.). Serum PSA level data were analyzed by Dunnett's test, which determines the significance of the effect of the different treatments. The therapeutic effect was further verified using the Fit model to test the effect of the different treatments during the experimental period. Where indicated, comparisons between 2 different individual treatments were performed using a 1-way ANOVA test (8). Survival experiments were analyzed using the Cox-Mantel Rank test.

\section{Acknowledgments}

The devoted assistance of Tami Danon, Sara Barak, and the team from the Endocrine Laboratory at Sheba Medical Center is gratefully acknowledged. We are grateful to the Prostate Cancer Foundation, the US Army, and the European Union for their support. This study was supported by an award from the CaP-CURE Foundation and grants from the US Army Prostate Cancer Research Program (DAMD17-98-1-8507) and the European Commission Quality of Life Program (QLK3-1999-01262).

Received for publication May 28, 2004, and accepted in revised form October 6, 2004.

Address correspondence to: Zelig Eshhar, Department of Immunology, The Weizmann Institute of Science, Rehovot 76100, Israel. Phone: 972-8-9344014; Fax: 972-8-9474030; E-mail: zelig.eshhar@weizmann.ac.il.
6. Ma, Q.Z., Gonzalo-Daganzo, R., and Junghans, R.P. 2001. Genetically engineered $T$ cells as adoptive immunotherapy of cancer. In Cancer chemotherapy o biological response modifiers, vol. 20. G. Giacone, R. Schilsky, and P. Sond, editors. Elsevier Science. Oxford, United Kingdom. 319-345.

7. Sadelain, M., Riviere, I., and Brentjens, R. 2003. Targeting tumours with genetically enhanced T lymphocytes. Nat. Rev. Cancer. 3:35-45.

8. Pinthus, J.H., et al. 2003. Immuno-gene therapy of established prostate tumors using chimeric receptor-redirected human lymphocytes. Cancer Res. 63:2470-2476

9. Campbell,J.J., and Butcher, E.C. 2000. Chemokines in tissue-specific and microenvironment-specific lymphocyte homing. Curr. Opin. Immunol. 12:336-341.

10. Kershaw, M.H., et al. 2000. Generation of genemodified $\mathrm{T}$ cells reactive against the angiogenic kinase insert domain-containing receptor (KDR) found on tumor vasculature. Hum. Gene Ther. 11:2445-2452.

11. Brentjens, R.J., et al. 2003. Eradication of systemic B-cell tumors by genetically targeted human T lymphocytes co-stimulated by CD80 and interleukin15. Nat. Med. 9:279-286.

12. Cashman, J.D., et al. 1997. Kinetic evidence of the regeneration of multilineage hematopoiesis from primitive cells in normal human bone marrow transplanted into immunodeficient mice. Blood. 89:4307-4316.

13. Kollet, O., et al. 2001. Rapid and efficient hom-

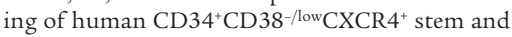
progenitor cells to the bone marrow and spleen of NOD/SCID and NOD/SCID/B2 $\mathrm{m}^{\text {null }}$ mice. Blood. 97:3283-3291.

14. Lapidot, T., and Kollet, O. 2002. The essential roles of the chemokine SDF-1 and its receptor CXCR4 in human stem cell homing and repopulation of transplanted immune-deficient 
NOD/SCID and NOD/SCID/B2 $\mathrm{m}^{\text {null }}$ mice. Lenkemia. 16:1992-2003.

15. Ponomaryov, T., et al. 2000. Induction of the chemokine stromal-derived factor-1 following DNA damage improves human stem cell function. J. Clin. Invest. 106:1331-1339.

16. Bleul, C.C., Fuhlbrigge, R.C., Casasnovas, J.M., Aiuti, A., and Springer, T.A. 1996. A highly efficacious lymphocyte chemoattractant, stromal cell-derived factor 1 (SDF-1). J. Exp. Med. 184:1101-1109.

17. Beider, K., et al. 2003. Involvement of CXCR4 and IL-2 in the homing and retention of human NK and NK T cells to the bone marrow and spleen of NOD/SCID mice. Blood. 102:1951-1958.

18. Kantele, J.M., Kurk, S., and Jutila, M.A. 2000. Effects of continuous exposure to stromal cellderived factor- 1 alpha on $\mathrm{T}$ cell rolling and tight adhesion to monolayers of activated endothelial cells. J. Immunol. 164:5035-5040.

19. Nanki, T., et al. 2000. Stromal cell-derived factor-1CXC chemokine receptor 4 interactions play a central role in CD4+ $\mathrm{T}$ cell accumulation in rheumatoid arthritis synovium. J. Immunol. 165:6590-6598.

20. Eshhar, Z., Waks, T., Bendavid, A., and Schindler, D.G. 2001. Functional expression of chimeric receptor genes in human T cells. J. Immunol. Methods. 248:67-76.

21. Jourdan, P., et al. 1998. IL-4 induces functional cellsurface expression of CXCR4 on human T cells. J. Immunol. 160:4153-4157.

22. Shirozu, M., et al. 1995. Structure and chromosomal localization of the human stromal cell-derived factor 1 (SDF1) gene. Genomics. 28:495-500.

23. Peled, A., et al. 1999. Dependence of human stem cell engraftment and repopulation of NOD/SCID mice on CXCR4. Science. 283:845-848.

24. Beecham, E.J., Ortiz-Pujols, S., and Junghans, R.P. 2000. Dynamics of tumor cell killing by human $\mathrm{T}$ lymphocytes armed with an anti-carcinoembryonic antigen chimeric immunoglobulin T-cell receptor. J. Immunother. 23:332-343.

25. Haynes, N.M., et al. 2001. Redirecting mouse CTL against colon carcinoma: superior signaling efficacy of single-chain variable domain chimeras containing TCR-zeta vs Fc epsilon RI-gamma. J. Immunol. 166:182-187.

26. Jensen, M., Tan, G., Forman, S., Wu, A.M., and Raubitschek, A. 1998. CD20 is a molecular target for $\mathrm{scFvFc}$ :zeta receptor redirected T cells: implications for cellular immunotherapy of CD20+ malignancy. Biol. Blood Marrow Transplant. 4:7583.

27. Hombach, A., et al. 1998. An anti-CD30 chimeric receptor that mediates $\mathrm{CD} 3$-zeta-independent T-cell activation against Hodgkin's lymphoma cells in the presence of soluble CD30. Cancer Res. 58:1116-1119.

28. Finney, H.M., Lawson, A.D., Bebbington, C.R., and Weir, A.N. 1998. Chimeric receptors providing both primary and costimulatory signaling in T cells from a single gene product. J. Immunol. 161:2791-2797.

29. Hekele, A., et al. 1996. Growth retardation of tumors by adoptive transfer of cytotoxic $\mathrm{T}$ lymphocytes reprogrammed by CD $44 \mathrm{v} 6$-specific scFv: zeta-chimera. Int. J. Cancer. 68:232-238.

30. Daly, T., et al. 2000. Recognition of human colon cancer by $T$ cells transduced with a chimeric receptor gene. Cancer Gene Ther. 7:284-291.

31. Ren-Heidenreich, L., Hayman, G.T., and Trevor, K.T. 2000. Specific targeting of EGP-2+ tumor cells by primary lymphocytes modified with chimeric T cell receptors. Hum. Gene Ther. 11:9-19.

32. Hwu, P., et al. 1993. Lysis of ovarian cancer cells by human lymphocytes redirected with a chimeric gene composed of an antibody variable region and the Fc receptor gamma chain. J. Exp. Med. 178:361-366.

33. Weijtens, M.E., Willemsen, R.A., Valerio, D., Stam, K., and Bolhuis, R.L. 1996. Single chain Ig/gamma gene-redirected human $\mathrm{T}$ lymphocytes produce cytokines, specifically lyse tumor cells, and recycle lytic capacity. J. Immunol. 157:836-843.

34. Weijtens, M.E., Willemsen, R.A., Hart, E.H., and Bolhuis, R.L. 1998. A retroviral vector system 'STITCH' in combination with an optimized single chain antibody chimeric receptor gene structure allows efficient gene transduction and expression in human T lymphocytes. Gene Ther. 5:1195-1203.

35. Yun, C.O., Nolan, K.F., Beecham, E.J., Reisfeld, R.A., and Junghans, R.P. 2000. Targeting of T lymphocytes to melanoma cells through chimeric antiGD3 immunoglobulin T-cell receptors. Neoplasia. 2:449-459.

36. Reinhold, U., et al. 1999. Specific lysis of melanoma cells by receptor grafted $\mathrm{T}$ cells is enhanced by anti-idiotypic monoclonal antibodies directed to the $\mathrm{scFv}$ domain of the receptor. J. Invest. Dermatol. 112:744-750.

37. Mezzanzanica, D., et al. 1998. Transfer of chimeric receptor gene made of variable regions of tumorspecific antibody confers anticarbohydrate specificity on T cells. Cancer Gene Ther. 5:401-407.

38. Willemsen, R.A., et al. 2000. Grafting primary human $\mathrm{T}$ lymphocytes with cancer-specific chimeric single chain and two chain TCR. Gene Ther. 7:1369-1377.

39. Gong, M.C., et al. 1999. Cancer patient T cells genetically targeted to prostate-specific membrane antigen specifically lyse prostate cancer cells and release cytokines in response to prostate-specific membrane antigen. Neoplasia. 1:123-127.

40. Bander, N.H., et al. 1997. MHC class I and II expression in prostate carcinoma and modulation by interferon-alpha and -gamma. Prostate. 33:233-239.

41. Sanda, M.G., et al. 1995. Molecular characterization of defective antigen processing in human prostate cancer. J. Natl. Cancer Inst. 87:280-285.

42. Croft, M. 2003. Costimulation of T cells by OX40, 4-1BB, and CD27. Cytokine Growth Factor Rev. 14:265-273.

43. Maus, M.V., et al. 2002. Ex vivo expansion of polyclonal and antigen-specific cytotoxic $\mathrm{T}$ lymphocytes by artificial APCs expressing ligands for the T-cell receptor, CD28 and 4-1BB. Nat. Biotechnol. 20:143-148.

44. Rosenberg, S.A., et al. 1994. Treatment of patients with metastatic melanoma with autologous tumorinfiltrating lymphocytes and interleukin 2. J. Natl. Cancer Inst. 86:1159-1166.

45. Yee, C., et al. 2000. Melanocyte destruction after antigen-specific immunotherapy of melanoma: direct evidence of T cell-mediated vitiligo. J. Exp. Med. 192:1637-1644.

46. Dudley, M.E., et al. 2001. Adoptive transfer of cloned melanoma-reactive $\mathrm{T}$ lymphocytes for the treatment of patients with metastatic melanoma. J. Immunother. 24:363-373.

47. Kjaergaard, J., et al. 2000. Biodistribution and tumor localization of lymphokine-activated killer $\mathrm{T}$ cells following different routes of administration into tumor-bearing animals. Cancer Immunol. Immunother. 48:550-560.

48. Rossi, D., and Zlotnik, A. 2000. The biology of chemokines and their receptors. Annu. Rev. Immunol. 18:217-242.

49. Liu, H., et al. 1997. Monoclonal antibodies to the extracellular domain of prostate-specific membrane antigen also react with tumor vascular endothelium. Cancer Res. 57:3629-3634.
50. Reiter, R.E., et al. 1998. Prostate stem cell antigen: a cell surface marker overexpressed in prostate cancer. Proc. Natl. Acad. Sci. U. S. A. 95:1735-1740.

51. Su, Z.Z., et al. 1996. Surface-epitope masking and expression cloning identifies the human prostate carcinoma tumor antigen gene PCTA-1 a member of the galectin gene family. Proc. Natl. Acad. Sci. U. S. A. 93:7252-7257.

52. Hubert, R.S., et al. 1999. STEAP: a prostate-specific cell-surface antigen highly expressed in human prostate tumors. Proc. Natl. Acad. Sci. U. S. A. 96:14523-14528.

53. Lyne, J.C., et al. 1997. Tissue expression of neu differentiation factor/heregulin and its receptor complex in prostate cancer and its biologic effects on prostate cancer cells in vitro. Cancer J. Sci. Am. 3:21-30.

54. Craft, N., et al. 1999. Evidence for clonal outgrowth of androgen-independent prostate cancer cells from androgen-dependent tumors through a twostep process. Cancer Res. 59:5030-5036.

55. Signoretti, S., et al. 2000. Her-2-neu expression and progression toward androgen independence in human prostate cancer. J. Natl. Cancer Inst. 92:1918-1925.

56. Corey, E., et al. 2003. LuCaP 35: a new model of prostate cancer progression to androgen independence. Prostate. 55:239-246.

57. Gomez, G.G., Hutchison, R.B., and Kruse, C.A. 2001. Chemo-immunotherapy and chemo-adoptive immunotherapy of cancer. Cancer Treat. Rev. 27:375-402.

58. Ganss, R., Ryschich, E., Klar, E., Arnold, B., and Hammerling, G.J. 2002. Combination of T-cell therapy and trigger of inflammation induces remodeling of the vasculature and tumor eradication. Cancer Res. 62:1462-1470.

59. Dunussi-Joannopoulos, K., et al. 2002. Efficacious immunomodulatory activity of the chemokine stromal cell-derived factor 1 (SDF-1): local secretion of SDF-1 at the tumor site serves as T-cell chemoattractant and mediates T-cell-dependent antitumor responses. Blood. 100:1551-1558.

60. Dudley, M.E., et al. 2002. Cancer regression and autoimmunity in patients after clonal repopulation with antitumor lymphocytes. Science. 298:850-854.

61. Geminder, H., et al. 2001. A possible role for CXCR4 and its ligand, the CXC chemokine stromal cell-derived factor-1, in the development of bone marrow metastases in neuroblastoma. J. Immunol. 167:4747-4757.

62. Libura, J., et al. 2002. CXCR4-SDF-1 signaling is active in rhabdomyosarcoma cells and regulates locomotion, chemotaxis, and adhesion. Blood. 100:2597-2606.

63. Muller, A., et al. 2001. Involvement of chemokine receptors in breast cancer metastasis. Nature. 410:50-56.

64. Zeelenberg, I.S., Ruuls-Van Stalle, L., and Roos, E. 2003. The chemokine receptor CXCR4 is required for outgrowth of colon carcinoma micrometastases. Cancer Res. 63:3833-3839.

65. Corey, E., et al. 2002. Establishment and characterization of osseous prostate cancer models: intratibial injection of human prostate cancer cells. Prostate. 52:20-33.

66. Stancovski, I., et al. 1991. Mechanistic aspects of the opposing effects of monoclonal antibodies to the ERBB2 receptor on tumor growth. Proc. Natl. Acad. Sci. U. S. A. 88:8691-8695.

67. Jung, S., et al. 2002. In vivo depletion of CD11 $\mathrm{c}^{+}$ dendritic cells abrogates priming of $\mathrm{CD}^{+} \mathrm{T}$ cells by exogenous cell-associated antigens. Immunity. 17:211-220. 